\title{
Evaluating the results of clinical trials in inflammatory bowel disease: Can we reconcile the controversies?
}

\author{
GARY R MAY MD FRCPC, ELDON A SHAFFER MD FRCPC FACP
}

A S CLINICIANS, WE ARE FACED WITH A LARGE BODY OF literature concerning the clinical evaluation of 'newer' medical therapies. In recent years, multiple pharmacological agents, including immunosuppressives, new delivery systems for aminosalicylic acid (5-ASA), more potent corticosteroids, and miscellaneous therapies, such as metronidazole and nutritional therapy, have been evaluated for inflammatory bowel disease (1). The results of trials evaluating these therapies are often equivocal, sometimes conflicting, and thus difficult to interpret. Extrapolating the results of positive clinical trials to patients in practice is not always easy (2). The reality of our limited health care resources have brought economic factors into consideration when evaluating medical or surgical therapy. More and more the question to be answered is not what is the most efficacious therapy, but rather which therapy will provide the best clinical outcome for the dollars spent. This means that in addition to trying to decide if the results of a trial are clinically significant, we must also consider whether the increased costs that are often involved with new therapies warrant their use over less expensive (and perhaps slightly less efficacious) ones. In this light, decision analyses and cost-effective analyses are now flourishing. Neither has yet to be applied to the management of inflammatory bowel disease.

A more critical appraisal of the published reports is essential to better apply their results to clinical practice. The perceptive articles by Sutherland (Can J Gastroenterol 1993;7:557-560) and Irvine (Can J Gastroenterol 1993;7:561567 ) focus on this issue. Methodologically sound studies with clinically relevant end-points should have the greatest influence over our therapeutic decisions.

Department of Medicine, Division of Gastroenterology, University of Calgary, Calgary, Alberta

Correspondence: Dr Gary R May, Division of Gastroenterology, University of Calgary, 3330 Hospital Drive NW, Calgary, Alberta

T2N 4N1. Telephone (403) 220-4557, Fax (403) 283-3028
The quality of clinical trials has come under greater scrutiny in recent years. Scoring systems, such as the one developed by Chalmers et al (3), provide a formal evaluation of the quality of reported clinical trials. This system assesses a clinical trial on the basis of design (eg, blinding, patient selection, use of placebos), the appropriate use of statistics and how the results were reported. Although not for routine use, applying such a scoring system to a group of studies allows a more critical perspective. For example, the assessment of a group of trials evaluating 5-ASA for treatment of ulcerative colitis showed that in many cases the reason studies lost points (had a lower rating) was due to the omission of details of the study design and statistics from the published report, even though the study was, in most cases, appropriately performed (4). The message here is that clinical investigators can improve the quality of their reported trials by making sure that key information regarding the design and statistics of their trial are included in the manuscript. The recent move in many fields to apply more clinically oriented outcome measures to clinical trials is an important step forwards and can only serve to improve their relevance. The McMaster Inflammatory Bowel Disease Questionnaire has been an important advance in the area of inflammatory bowel disease (5).

Even with well done clinical trials, we are often faced with several small negative studies or conflicting results between studies. How are we to reconcile these differences? One technique that increasingly is being used is meta-analysis. Metaanalysis is a formal technique of literature review that allows similar trials to be combined and the results pooled. It is most powerful when the findings of several studies are inconclusive but individually lack sufficient power to answer a specific question or when studies have conflicting results $(6,7)$. Although initially developed to be applied to randomized controlled trials, methodology is evolving to apply similar techniques to cohort and case-control studies $(8,9)$. The basic feature of any meta-analysis is that, just like a clinical trial, a formal protocol is followed to answer a specific question. 
Inclusion and exclusion criteria are set a priori and then applied to a group of studies. The literature review must be extensive and complete as the findings of missed studies can bias the results. A quality analysis usually is done as part of the analysis. Once the studies are selected, the results are formally extracted and outcome measures determined. The results are then combined by a variety of statistical methods. Most of these methods give extra weight to larger studies. One criticism of meta-analysis is that it may combine 'apples and oranges'. Although there always will be variation between trials, meta-analysis should only combine those studies that are reasonably similar and should highlight any excessive variation among the studies.

When properly applied and executed, meta-analysis can be a powerful tool. As an example, we recently completed a meta-analysis of 5-ASA therapy for acute and maintenance therapy of ulcerative colitis (10). Despite the fact that no single study has demonstrated superiority of the newer 5-ASA preparations over sulphasalazine, these products have largely replaced sulphasalazine in Canada; this is associated with a significant increase in cost as most of the newer preparations are two to three times more expensive than sulphasalazine. Our meta-analysis confirmed that although the newer 5-ASA products were superior to placebo, they were merely equivalent in efficacy to sulphasalazine. Given the cost considerations, it is still reasonable to recommend sulphasalazine in the $80 \%$ of patients who are able to tolerate this medication. Such a recommendation can be made with greater confidence based upon the pooled results of the meta-analysis than upon any of the single studies. Indeed, it would be difficult to make such a recommendation based upon a single study with a low power of detecting a small difference between two similar drugs.

Clinical evaluation of inflammatory bowel disease remains difficult and challenging. It is difficult to predict what the future holds. Novel approaches, such as searching for genomes associated with inflammatory bowel disease, are major undertakings with unclear benefits. Yet, 10 years ago cystic fibrosis had no potential cure and therapy was sympto- matic and palliative. Identification of the cystic fibrosis gene and the potential for gene therapy has made the possibility of a 'cure' for cystic fibrosis a reality. Until such a 'cure' for inflammatory bowel disease is found, the therapeutic questions facing us entail comparing therapies that are only marginally different in terms of efficacy. Outcome measures beyond disease activity indices will be more important in the future. Economic issues must be addressed. Even with rigorous clinical trial methodology, one can anticipate equivocal results as the questions being addressed are increasingly complex. By using the results of only well done, clinically applicable trials and applying techniques such as meta-analysis when appropriate, we should be able to better reconcile some of the controversy.

\section{REFERENCES}

1. Peppercorn MA. Advances in drug therapy for inflammatory bowel disease. Ann Intern Med 1990;112:50-60.

2. Kirsner JB. Limitations in the evaluation of inflammatory bowel disease: Suggestions for future research. J Clin Gastroenterol 1990;12:516-24.

3. Chalmers TC, Smith H Jr, Blackburn B, et al. A method for assessing the quality of a randomized control trial. Cont Clin Trial 1981;2:31-49.

4. Sutherland LR, May GR. Errors of omission or commission: Improving the reporting of clinical trials. Gastroenterology 1993;104:A786. (Abst)

5. Guyatt GH, Mitchell A, Irvine EJ, et al. A new measure of health status for clinical trials in inflammatory bowel disease. Gastroenterology 1989;96:804-10.

6. Sacks HS, Berrier J, Reitman D, Ancona-Berk VA, Chalmers TC. Meta-analyses of randomized controlled trials. N Engl J Med 1987;316:450-5.

7. L'Abbe KA, Detsky AS, O'Rourke K. Meta-analysis in clinical research. Ann Intern Med 1987;107:224-33.

8. Greenland S. Quantitative methods in the review of epidemiologic literature. Epidemiol Rev 1987;9:1-30.

9. Giovannucci E, Colditz GA, Stampfer MJ. A meta-analysis of cholecystectomy and risk of colorectal cancer. Gastroenterology 1993;105:130-41.

10. Sutherland LR, May GR, Shaffer EA. Sulfasalazine revisited: A meta-analysis of 5-aminosalicylic acid in the treatment of ulcerative colitis. Ann Intern Med 1993;118:540-9. 


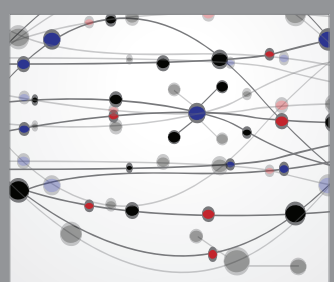

The Scientific World Journal
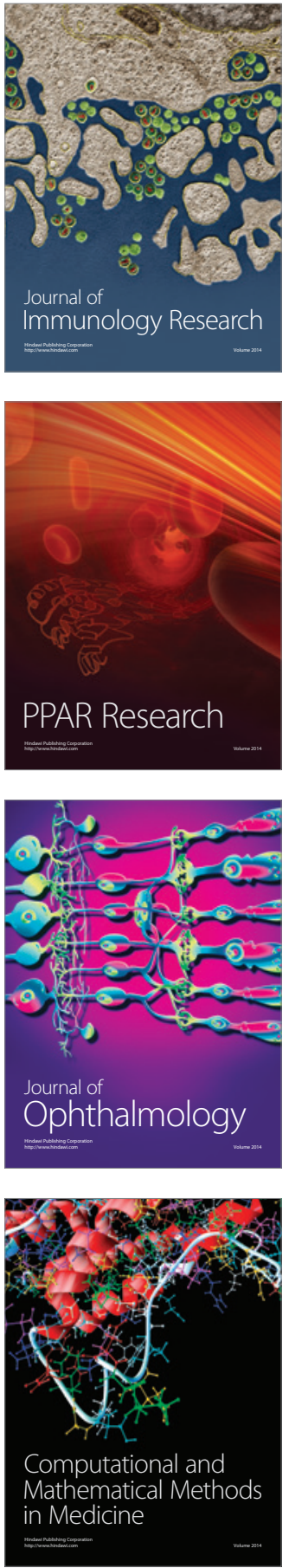

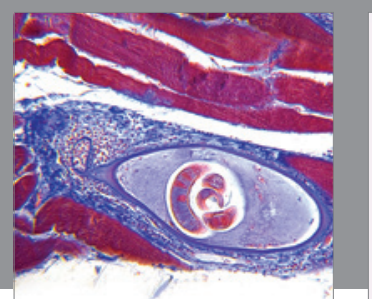

Gastroenterology Research and Practice

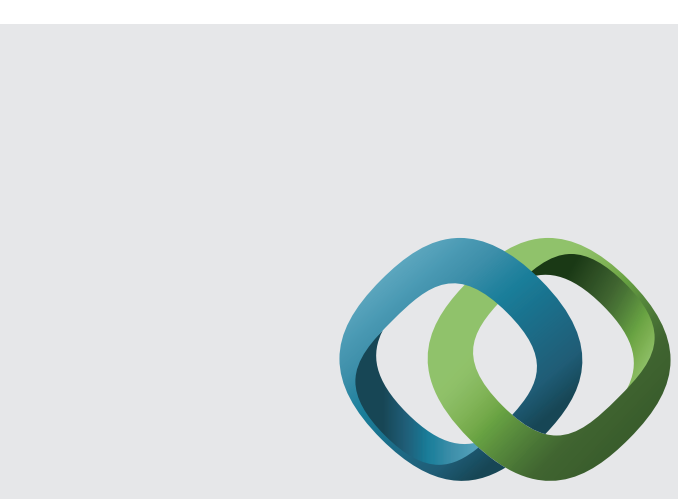

\section{Hindawi}

Submit your manuscripts at

http://www.hindawi.com
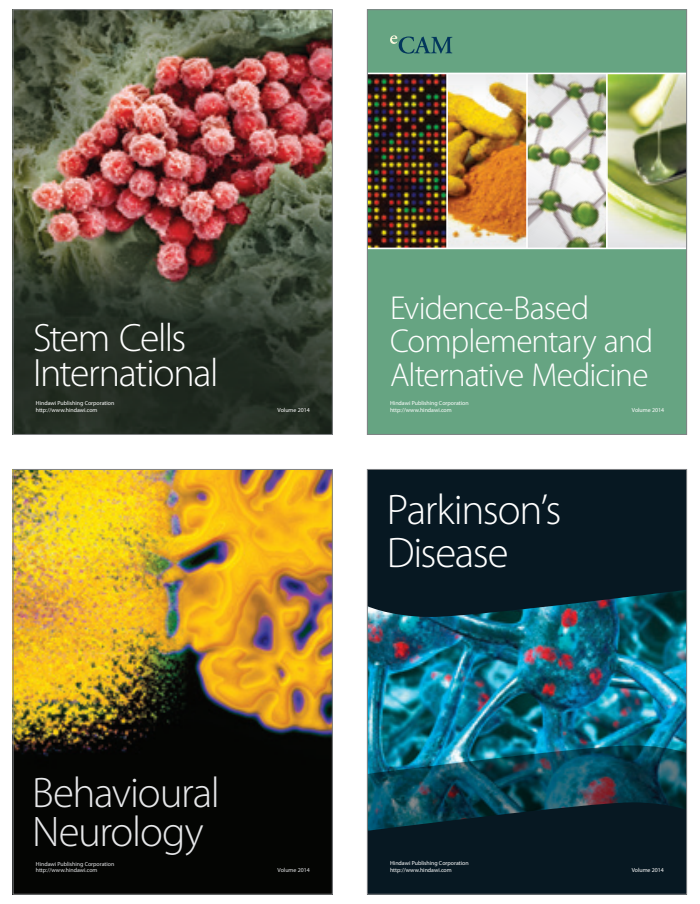
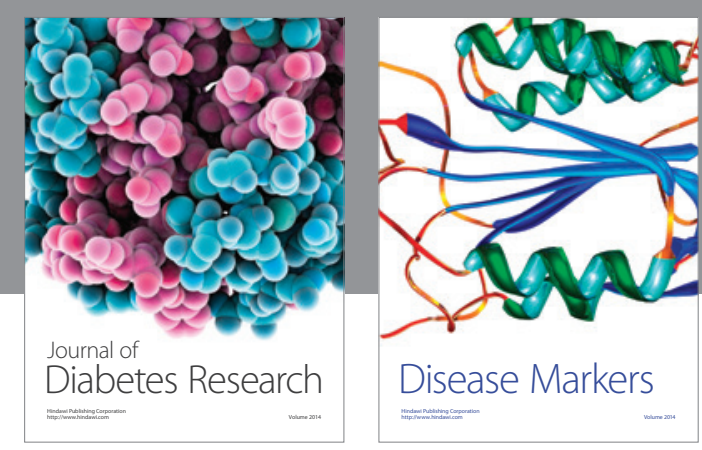

Disease Markers
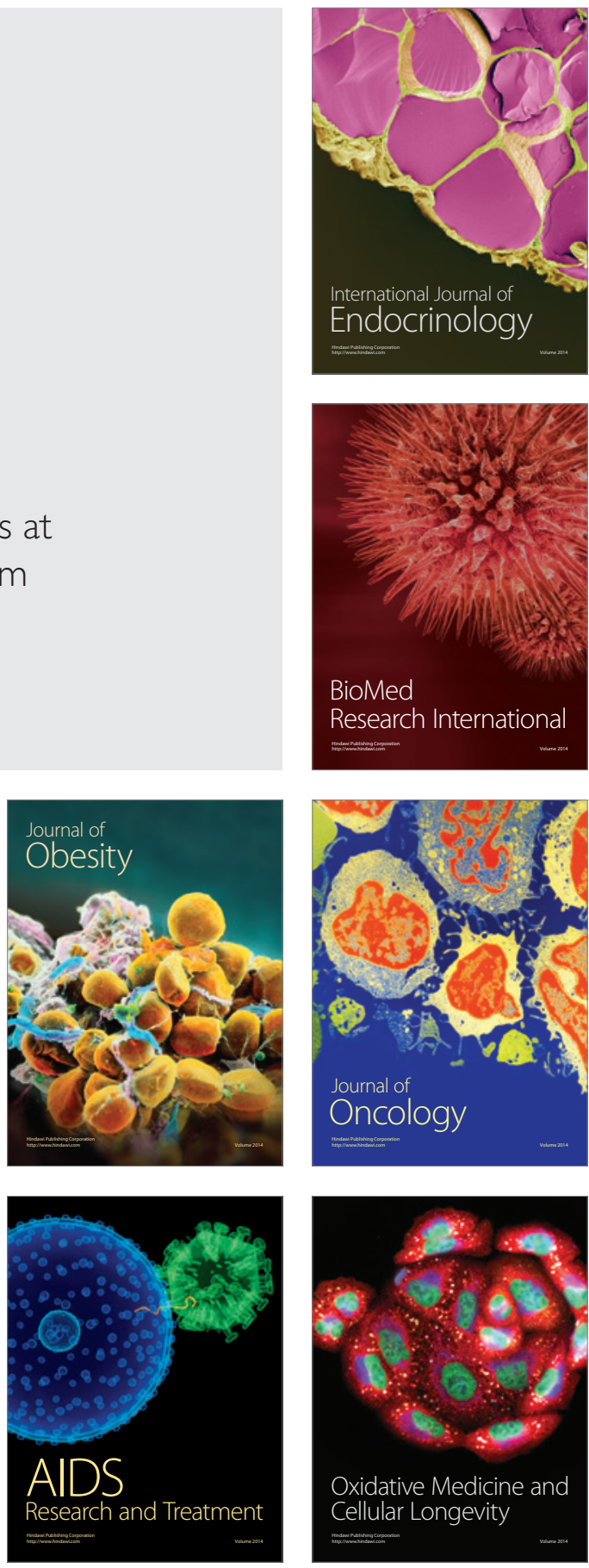